\title{
Modulation of the equine microbiome by pasture and feed supplements: A metabolomics approach
}

\author{
Snalune $\mathrm{KL}^{1}$, Hunter $\mathrm{JO}^{2}$, Waring $\mathrm{RH}^{3 *}$, Cauchi $\mathrm{M}^{4}$, Turner $\mathrm{C}^{5}$ and Batty $\mathrm{C}^{5}$ \\ ${ }^{1}$ MRCVS, Newnham Court Equine Clinic, Maidstone Kent, ME14 5E, UK \\ ${ }^{2}$ FRCP, Gastroenterology Research Unit, Addenbrooke's Hospital, Cambridge, CB2 0QQ, UK \\ ${ }^{3} \mathrm{DSc}$, School of Biosciences, University of Birmingham, Birmingham, B15 2TT, UK \\ ${ }^{4} \mathrm{PhD}$, Department of Mathematics and Statistics, University of Limerick, Limerick, V94T9PX, Ireland \\ ${ }^{5} \mathrm{PhD}$, Department of Chemistry/Analytical Sciences, Open University, Milton Keynes, MK7 6AA, UK
}

\begin{abstract}
Faecal VOCs (volatile organic compounds) were compared in horses and ponies before and after 6 weeks on Spring pasture and with and without a dietary supplement (ERME). The supplement was derived from malted barley and contained enzymes which degrade complex carbohydrates, including amylase, maltase, glucanases and fructanases. VOCs of faecal samples were analysed by selected ion flow tube mass spectrometry (SIFT-MS) and the results showed that the faecal microbiome was altered in different ways both by feeding on Spring pasture and by the dietary supplement. The composition of the equine gut microbiome (EGM) is therefore potentially a reflection of dietary constituents, both pasture and supplements; any metabolomic approach should take this into account since the EGM may have modulating effects on physiological parameters such as diabetes and obesity.
\end{abstract}

\section{Introduction}

Metabolomics is a powerful technique which allows complex assessments of the relative importance of multiple metabolites detected in a biofluid or tissue. The volatile faecal metabolome is defined as the array of volatile metabolites of low molecular weight found in the headspace above a faecal sample and can be analysed using techniques such as gas or liquid chromatography or nuclear-magnetic resonance (NMR) combined with mass spectrometry. Metabolic profiling captures information from both intrinsic (genetics, protein expression) and environmental inputs (diet, gut microbiota) providing an overview of the effects of multiple factors that may be acting on the organism. This technique has proven highly effective for unravelling complex metabolic interactions between the mammalian host and its resident gut microbiota [1-4].

Volatile organic compounds (VOCs) in body fluids have been shown to vary in human disease, and changes in faecal VOCs are used as surrogate markers for alterations in the gut microbiome. In humans, this may vary with diet but relatively little is known about the equivalent equine gut microbiome (EGM). Horses are often kept on fixed rations, with the addition of varying amounts of commercially-available nutrients. This study was performed to investigate the VOCs found in the faecal metabolome of pastured horses/ponies and to determine whether they can be modulated by changes in pasture or addition of enzyme-rich dietary supplements. An intervention study was therefore performed on 45 pastured animals at a sanctuary in Eastern England in Spring, at a time when grazing is known to change in character as new grass becomes available. A commercially-available malt-based enzymerich supplement (ERME), which contains a wide range of enzymes for degradation of complex carbohydrates, was also studied to determine its effect on the EGM.

\section{Methods}

\section{Study protocol}

Faecal samples were collected non-invasively either before and six weeks after supplementation with ERME while keeping the horses/ ponies on pasture for 6 weeks in Spring. The volatile faecal metabolome was characterised using selected ion flow tube-mass spectrometry (SIFT-MS) in conjunction with multivariate data analysis.

\section{Study population and sampling}

A study population of 45 horses and ponies at the Redwings Sanctuary, Norfolk was chosen by the resident veterinary officer to take part in the study. This was performed in Spring from early May to midJune, the timing being chosen so that the effects of eating new grass could be determined. A power calculation was performed based on previous data using ERME as a dietary supplement [4]. Based on a significance of $\mathrm{p}<0.05$ and a power of 0.8 , the sample size required was 18 and a minimum of 20 horses per group was randomly selected. The study population was chosen based on the following inclusion and exclusion criteria. Inclusion criteria included: $>2$ years old, no physical evidence of disease, based on a clinical examination by a veterinary officer, and resident at the sanctuary for $>1$ year. Exclusion criteria included being on any medication, pregnancy and no entire males. Horses were randomly allocated to either the treatment group or the control group.

${ }^{*}$ Correspondence to: Waring RH, DSc, School of Biosciences, University of Birmingham, Birmingham, B15 2TT, UK, Tel: 01214477881; E-mail: r.h.waring@bham.ac.uk

Received: January 24, 2019; Accepted: February 11, 2019; Published: February 13,2019 
There were 36 geldings and 9 mares between the ages of 4 and 23 years old. Breeds represented were wide-ranging including Shetland pony, Welsh pony, Cob types and Thoroughbred/Thoroughbred cross types. Horses remained under the care of the sanctuary throughout the study. All were allowed free access to grazing twenty-four hours a day. They were moved to a new pasture 7 days prior to starting the study and remained on this pasture throughout the entire study. There was no specific difference between pastures before and after commencement of the study. All horses were free from concurrent medical treatment for the duration of the study. All horses in the study population were assessed for body condition score and cresty neck score prior to supplementation and after six weeks supplementation. A $30 \mathrm{~g}$ faecal sample was collected once before supplementation and once after six weeks of twice-daily supplementation in feed. Sampling commenced approximately three hours after morning feeding and took approximately 3 hours on each occasion for all horses to pass faeces. Freshly voided faeces were collected into polyethylene sample containers and kept refrigerated before transport and transfer into a freezer at a temperature of $-80^{\circ} \mathrm{C}$. Horses were excluded from the study if they received any medication during the study or if they were unavailable for sampling. Sampling and metadata collection were carried out under University of Cambridge ethics approval CR83 with the informed consent of the sanctuary. The horses were randomly divided into two groups: 21 controls (group C) and 24 test animals (group D). All horses were fed a standard diet of 200 grams of a high fibre ration, Dengie Hi-Fi Lite, (DE 7.5 MJ/kg) with added vitamins and minerals, twice a day. The test group (D) was given in addition $300 \mathrm{mls}$ of ERME twice daily for six weeks and consisted of 24 horses and ponies ( 22 geldings, 2 females) aged 5 to 23 years old (median age 8 years). Group C (control group) consisted of 21 horses and ponies (15 geldings, 6 females) aged 4 to 18 years old (median age 7.5 years).

\section{Sample preparation}

Volatile organic compounds (VOCs) in the equine faecal headspace were measured using selected ion flow tube-mass spectrometry (SIFT-MS). Faecal samples were taken from the $-80^{\circ} \mathrm{C}$ freezer and thoroughly defrosted. Exactly $5 \mathrm{~g}$ of each sample was weighed out and placed into a sample bag constructed from Nalophan tubing ${ }^{c}$. The bag was filled with zero grade (hydrocarbon free) air before being sealed with a Swagelok fitting, then placed in the incubator at $40^{\circ} \mathrm{C}$ for 45 minutes to increase compound volatilization. After incubation, samples were attached to the SIFT-MS via the heated sampling capillary. The precursor ions were generated in an air/water mixture via a microwave discharge and then selected via an upstream quadrupole mass filter. Selected precursor ions $\left(\mathrm{H}_{3} \mathrm{O}^{+}, \mathrm{NO}^{+}\right.$ or $\mathrm{O}_{2}^{+}$) were then injected into helium carrier gas and passed along a flow tube into which the sample was introduced via a heated capillary. The product ions resulting from precursor ion/sample reaction were then separated in a downstream quadrupole mass filter before being detected and counted. After attaching the sample bag, the SIFT-MS tested each sample with the three precursor ions in turn: $\mathrm{H}_{3} \mathrm{O}^{+}, \mathrm{NO}^{+}$ and $\mathrm{O}_{2}{ }^{+}$. The spectrum was analysed from mass-to-charge ratio $(\mathrm{m} / \mathrm{z})$ 10 to 140 , the time in which each scan lasted being set at 6 cycles of 5 seconds for each ion. This procedure was repeated with every sample tested. Data were then transferred to the computer to be analysed for the range of possible compounds present in each sample.

\section{Multivariate data analysis}

The acquired data were pre-processed and analysed using Matlab R2011a (Mathworks Inc, USA) incorporating functions from the PLS
Toolbox v3.5 (Eigenvector Research Inc., USA). Exploratory data analysis using principal components analysis (PCA) was also employed to observe any trends and groupings within the respective datasets, and to identify any outlying samples. These were identified visually and via the Hotelling $\mathrm{T}^{2}$ test (a multivariate extension to the Student t-test). Once identified, they were removed. The effects of feature selection were also assessed by scanning the data for significantly different peaks $(\mathrm{m} / \mathrm{z}$ peaks for SIFT-MS). The following comparisons were made between:

- Groups C and D at week 0

- Groups C and D at week 6

- Group C at week 0 and week 6

\section{- Group D at week 0 and week 6}

The statistical methods employed were the parametric Student t-test and the non-parametric Wilcoxon T test.

Pattern recognition was performed by way of multivariate classification. Different modelling algorithms were assessed such as partial least squares discriminant analysis (PLS-DA) [5], support vector machines (SVMs) using radial basis function (RBF) and linear kernels and artificial neural networks (ANNs) via probabilistic neural networks [6]. The models were evaluated and optimised via crossmodel validation which incorporates bootstrapping with optimisation via leave-one-out cross validation (LOO-CV). In all, 150 evaluations were performed leading to the means of the accuracy (overall correctly classified: \%CC), specificity (\%SPEC), sensitivity (\%SENS) and the area under the receiver operating characteristic (AUROC) curve being reported. Permutation testing was also performed to ensure that the reported accuracies were indeed statistically significant, and not due to chance. The distributions of the two processes were visualised and assessment of the significance was calculated by the statistical z-test, which compares the means of two distributions (assumed to be normal). Accuracies (\%CC) closer to 50\% show less distinction between 2 groups, whereas accuracies (\%CC) closer to $100 \%$ show increasing distinction between 2 groups.

\section{Results}

Pastured horses and ponies (24) fed ERME were compared with controls (21). In the control group (C), body condition score (BCS), using the Henneke scale, was a median of $6 / 9$ at the start of the test period and 6/9 at the end. Cresty neck score (CNS) was a median of $2.5 / 5$ pre-test and $3 / 5$ post-test. In the experimental group (D), BCS was a median of $7 / 9$ pre-supplementation and 7/9 post-supplementation. CNS was a median of $3 / 5$ pre-supplementation and $3 / 5$ postsupplementation. One horse was removed from the control group, due to treatment for a corneal ulcer. All animals were assessed twice daily by sanctuary staff, trained in identifying common signs of disease. No animals developed laminitis or colic during the study. The volatile faecal metabolome was dominated by organic acids, alcohols and ketones and no significant differences could be distinguished between the two groups at the beginning of the study (60.5\%). However, marked changes were detected in both groups after 6 weeks, showing that both Spring pasture and ERME affected the volatile faecal metabolome, although in different ways. After 6 weeks on Spring pasture, group C (controls) had a markedly altered volatile metabolome (95.0\%) from that at week 0. Group D (test group fed ERME) also had a markedly altered volatile metabolome from that at week $0(78.7 \%)$. The two groups were significantly different from each other, suggesting the two groups were affected differently $(80.4 \%)$; the means of the two 
distributions were significantly different and thus not due to chance at the $95 \%$ confidence interval $(p<0.05)$. Table 1 shows the respective specificities, sensitivities and AUROC (area under the receiver operating characteristic) curve. Table 2 shows the influential $\mathrm{m} / \mathrm{z}$ ions identified from the PLS-DA loadings as likely to be contributing to the percent correctly classified along with the suggested compounds. Ions can arise from more than one compound (isotopologues); in this table, only the most likely tentative identifications of compounds are given. Both control and test groups show agreement in the form of propanol at $\mathrm{m} / \mathrm{z} 43$ (either 1-or 2-propanol which SIFT-MS currently cannot distinguish), methanol at $\mathrm{m} / \mathrm{z} 51$, acetone at $\mathrm{m} / \mathrm{z} 77$ and butyric acid at $\mathrm{m} / \mathrm{z} 107$. However, distinctive compounds are also suggested such as isoprene at $\mathrm{m} / \mathrm{z} 69$ (which may be present alongside methanol also at $\mathrm{m} / \mathrm{z} 69$-yet other compounds may also produce an ion at $\mathrm{m} / \mathrm{z} 69$ ), and the ion $\mathrm{m} / \mathrm{z} 93$ (which may be identified as toluene or propionic acid) in the Control group, and acetic acid at $\mathrm{m} / \mathrm{z} 61$ ), pentanoic acid or methyl butyrate both at $\mathrm{m} / \mathrm{z} 103$ in the Test group. The analysis has identified VOCs (e.g. propanol and methanol), which differentiate between horses and ponies pre- and post-supplementation. The median abundance for methanol, isoprene and acetone is significantly reduced after six weeks on Spring pasture, which indicates a potential change in fermentation processes in the gut. Abundances of methanol and acetone are also reduced post supplementation with ERME but the difference is not statistically significant.

\section{Discussion}

This study demonstrates that six weeks of Spring pasture and twice-daily supplementation with ERME both significantly alter the profile of VOCs of faeces from pastured horses/ponies, the VOCs being different in each case. The VOCs detected above the equine faecal headspace of pastured horses and ponies are dominated by acids and alcohols, as well as the apparent presence of esters, probably arising from bacterial digestion of carbohydrates, including dietary fibre [4]. The compounds identified as being significant are those expected in the headspace of faeces, and include the acids, alcohols and esters, but they are present at statistically significantly different concentrations in the test samples. There are therefore differences in the compounds present in the gut, and as these most likely arise from bacterial activity, this reflects changes in the relative amounts or proportions of gut bacteria. The overall classification accuracies (\%CC) in the Control and Test groups were $95.0 \%$ and $78.7 \%$ respectively. This difference strongly suggests that both Spring pasture and the application of ERME have altered the faecal metabolome. This is further supported by the comparison of the two groups at week 0 and at week 6 . At week $0(60.5 \%)$, prior to any dietary change, any differences could be attributed to inter-horse variation such as gender, age, prior pasture difference, weather, and other factors. At

Table 1. Comparison of control and test groups at week 0 and week 6

\begin{tabular}{|l|c|c|c|}
\hline Comparison & \% Correctly Classified & Specificity (\%) & Sensitivity (\%) \\
\hline Control vs Test group Week 0 & 60.5 & 54.00 & 66.2 \\
\hline Control vs Test group Week 6 & 80.4 & 85.2 & 76.2 \\
\hline Control Week 0 vs Week 6 & 95.0 & 91.6 & 0.6595 \\
\hline Test group Week 0 vs Week 6 & 78.7 & 74.8 & 0.8569 \\
\hline
\end{tabular}

"AUROC: Area under the receiver operating characteristic

Table 2. List of potential compounds pertaining to the $\mathrm{m} / \mathrm{z}$ ions deemed significant by the PLS-DA loadings $\left(\mathrm{H}_{3} \mathrm{O}^{+}\right.$precursor ions only)

\begin{tabular}{|c|c|c|}
\hline $\mathbf{m} / \mathbf{z}$ & $\begin{array}{c}\text { Possible Compounds } \\
\text { Control, week 0 vs week 6: } 95.0 \%\end{array}$ & $\begin{array}{c}\text { Difference in Median Abundance from Box \& } \\
\text { Whisker Plots }\end{array}$ \\
\hline 33 & Methanol & Decrease (3.5) \\
\hline 51 & Methanol & Decrease (4.0) \\
\hline 59 & Acetone & Decrease (2.0) \\
\hline 69 & Isoprene/Methanol/Furan/Pentanal/3-Methyl butanal & Decrease $(9.0)$ \\
\hline 77 & Acetone & Decrease (3.5) \\
\hline 93 & Toluene/Butanol/Dimethyl Ether/Propanoic Acid & Decrease $(0.5)$ \\
\hline 107 & Xylene/Pentanol/Benzaldehyde/Butyric Acid/Ethyl acetate & No Change \\
\hline \multicolumn{3}{|c|}{ Test, week 0 vs week $6: 78.7 \%$} \\
\hline 30 & Unknown & Increase (3.0) \\
\hline 43 & 1- or 2-propanol & Decrease $(0.5)$ \\
\hline 51 & Methanol & Decrease $(2.5)$ \\
\hline 56 & Unknown (Butanal at 55; Butanol at 57) & Increase $(0.5)$ \\
\hline 61 & Acetic acid/Propanol/Methyl formate & Decrease $(0.2)$ \\
\hline 77 & Acetone & Decrease (2.5) \\
\hline 103 & $\begin{array}{l}\text { Pentanoic acid/Valeric acid/Trimethyl acetic acid/Ethyl propionate/Methyl } \\
\text { Butyrate }\end{array}$ & No Change \\
\hline 107 & Xylene/Pentanol/Benzaldehyde/Butyric acid/Ethyl acetate & No Change \\
\hline \multicolumn{3}{|c|}{$\begin{array}{c}\text { Control vs Test } \\
\text { Week } 6: 80.4 \%\end{array}$} \\
\hline 31 & Formaldehyde & Increase $(0.2)$ \\
\hline 45 & Acetaldehyde & Decrease (1.0) \\
\hline 63 & Acetaldehyde & Decrease $(2.5)$ \\
\hline 69 & Methanol & Increase (1.0) \\
\hline 77 & Acetone & Increase $(2.0)$ \\
\hline 127 & Methyl phenol & No Change \\
\hline
\end{tabular}


week 6, ERME appears to have altered the faecal metabolome (80.4\%) which is now more consistent between the horses. Proudman et al. [4] used the same ERME supplement (which contains a wide range of enzymes hydrolyzing complex carbohydrates) and similarly found changes in volatile faecal VOCs in the thoroughbred racehorse. Diets high in readily-hydrolysable carbohydrate, often in the form of grain, are commonly employed to provide energy required for fast physical activity but are widely recognized to be associated with an increased risk of intestinal disease as pancreatic and small intestinal amylase production is known to be low in the horse compared with other species [7]. Exogenous dietary amylase has previously been shown to enhance equine carbohydrate digestion [8]. Proudman et al. [4] suggested that the changes in faecal metabolome associated with the use of ERME are potentially a response to enhanced pre-caecal digestion of dietary carbohydrate. This would reduce and alter the substrates available for fermentation in the caecum hence altering the microbiome. It is interesting to note that acetate fell significantly in the test group receiving ERME, but not in the controls. Again, this was similar to effects seen in thoroughbred racehorses [4] suggesting that ERME may allow better colonic absorption of SCFAs possibly by prolonging intestinal transit time. The faecal metabolome was also altered by the changes in grazing quality over the period of the trial. The non-structural carbohydrate (NSC)-content of pasture has both diurnal and seasonal variations [9] and is highest in late Spring, the same time of year as this study was undertaken [10]. This may explain the marked changes in VOCs detected over the 6-week period in the control group and is relevant to any development of laminitis which is known to be at least partly due to pasturage on Spring grass.

Faecal VOCs and VOC profiles have been found to be associated with several intestinal diseases in man and horses [11-14]. Equinespecific metabolomics studies include a plasma nuclear magnetic resonance (NMR) spectroscopy study using an oligofructose overload model of early time-point laminitis [13] and a study profiling the volatile faecal metabolome of horses with colic [15]. However, there is little data on the factors affecting the EGM of healthy non-thoroughbred horses on standard pasture. Current studies in humans suggest that the gut microbiome is involved in modulation of clinical conditions such as diabetes and obesity [16,17]; attempts to link the EGM with similar conditions in horses [18] will require careful control of diet and dietary components for meaningful results.

\section{Manufacturers' addresses}

${ }^{a}$ Muntons, Stowmarket, Suffolk, UK.

${ }^{b}$ Dengie Hi-fi, Essex, UK.

${ }^{c}$ Kalle, Germany.

\section{References}

1. Jacobs DM, Deltimple N, van Velzen E, van Dorsten FA, Bingham M, et al. (2008) (1) H NMR metabolite profiling of feces as a tool to assess the impact of nutrition on the human microbiome. NMR Biomed 21: 615-626. [Crossref]

2. Turnbaugh PJ, Gordon JI (2008) An invitation to the marriage of metagenomics and metabolomics. Cell 134: 708-713. [Crossref]

3. Escalona E, Leng J, Dona AC, Merrifield CA, Holmes CJ, et al, (2015) Dominant components of the Thoroughbred metabolome characterised by $1 \mathrm{H}$-nuclear magnetic resonance spectroscopy: A metabolite atlas of common biofluids. Equine Vet J 47: 721 730. [Crossref]

4. Proudman CJ, Hunter JO, Darby AC, Escalona E, Batty C, et al. (2015) Characterisation of the faecal metabolome and microbiome of Thoroughbred racehorses. Equine Vet $J$ 47: 580-586. [Crossref]

5. Barker M, Rayens W (2003) Partial least squares for discrimination. J Chemom 17: 166-173.

6. Hagan MT, Demuth HB, Beale M (1996) Neural network design. International Thompson Publishing, Boston.

7. Kienzle E, Radicke E, Landes E, Kleffken D, Illenseer M, et al. (1994) Activity of amylase in the gastrointestinal tract of the horse. J Anim Physiol Anim Nutr 72: 234241 .

8. Richards N, Choct T, Hinch GN, Rowe JB (2004) Examination of the use of exogenous $\alpha$-amylase and amyloglucosidase to enhance starch digestion in the small intestine of the horse. Anim Feed Sci Technol 114: 295-305

9. Longland AC, Cairns AJ, Humphreys MO (1999) Seasonal and diurnal changes in fructan concentration in Lolium perenne: Implications for the grazing management of equines predisposed to laminitis. Proceedings of the 16th equine nutrition and physiology society symposium, June 2-5, Raleigh, NC. pp: 258-259.

10. Longland AC, Byrd BM (2006) Pasture nonstructural carbohydrates and equine laminitis. J Nutr 136: 2099S-2102S. [Crossref]

11. Cauchi M, Fowler DP, Walton C, Turner C, Jia W, et al. (2014) Application of gas chromatography mass spectrometry (GC-MS) in conjunction with multivariate classification for the diagnosis of gastrointestinal diseases. Metabolomics 10: $1113-$ 1120 .

12. Walton C, Fowler DP, Turner C, Jia W, Whitehead RN, et al. (2013) Analysis of volatile organic compounds of bacterial origin in chronic gastrointestinal diseases. Inflamm Bowel Dis 19: 2069-2078. [Crossref]

13. Keller MD, Pollitt CC, Marx UC (2011) Nuclear magnetic resonance-based metabonomic study of early time point laminitis in an oligofructose-overload model. Equine Vet $J$ 43: 737-743. [Crossref]

14. Probert CS, Reade S, Ahmed I (2014) Faecal volatile organic compounds: A novel, cheaper method of diagnosing IBD? Expert Rev Clin Immunol 10: 1129-1131. [Crossref]

15. Turner C, Batty C, Escalona E, Hunter JO, Proudman C (2013) The use of SIFT-MS in profiling the faecal volatile metabolome in horses with colic: Pilot study. Curr Anal Chem 9: 614-621.

16. Cani PD (2018) Human gut microbiome: Hopes, threats and promises. Gut 67: 17161725. [Crossref]

17. Meijnikman AS, Gerdes VE, Nieuwdorp M, Herrema H (2018) Evaluating causality of gut microbiota in obesity and diabetes in humans. Endocr Rev 39: 133-153. [Crossref]

18. Mshelia ES, Adamu L, Wakil Y, Turaki UA, Gulani IA, et al. (2018) The association between gut microbiome, sex, age, and body condition scores of horses in Maiduguri and its environs. Microb Pathog 118: 81-86. [Crossref]

Copyright: (C2019 Snalune KL. This is an open-access article distributed under the terms of the Creative Commons Attribution License, which permits unrestricted use, distribution, and reproduction in any medium, provided the original author and source are credited. 